\title{
Continuous Flow Fast Atom Bombardment with Packed Microcolumns: A Comparison of Precolumn Versus Coaxial Matrix Delivery
}

\author{
Stephen Pleasance and Pierre Thibault \\ Atlantic Research Laboratory, National Research Council of Canada, Halifax, Nova Scotia, Canada
}

\author{
M. Arthur Moseley,* Leesa J. Deterding, and Kenneth B. Tomer \\ Laboratory of Molecular Biophysics, National Institute of Environmental Health Sciences, Research Triangle \\ Park, North Carolina, USA
}

J. W. Jorgenson

Department of Chemistry, University of North Carolina at Chapel Hill, Chapel Hill, North Carolina, USA

The effect of adding glycerol to the mobile phase on the chromatographic separation of peptides has been investigated using a continuous flow fast atom bombardment (CFFAB) interface coupled with commercial packed microcolumns $(25 \mathrm{~cm} \times 320 \mu \mathrm{m}$ i.d.). In a comparative study using a UV detector, it was found that chromatographic peak broadening progressively increased with increasing percentage of glycerol in the mobile phase. In the liquid chromatographic FAB mass spectrometric analysis, this effect is compounded by the dynamic mixing of the column effluent on the probe. Improvements of $25-155 \%$ in the overall separation efficiencies were obtained by introducing the matrix independently to the probe tip via a coaxial arrangement. Application of this coaxial CFFAB is demonstrated by the analysis of peptide mixtures and tryptic digests. (J Am Soc Mass Spectrom 1990, 1, 312-319)

$\mathrm{O}$ ver the past decade, numerous liquid chromatography combined with mass spectrometry (LC/MS) interfaces using conventional (4.6 $\mathrm{mm}$ i.d.) and microbore (1.0-2.0 $\mathrm{mm}$ i.d.) columns have been developed, and the literature up to the end of 1985 has been reviewed in detail [1-5]. In view of the practical limits imposed by the vacuum system of the mass spectrometer, a maximum liquid flow rate of approximately $10 \mu \mathrm{L} / \mathrm{min}$ can generally be tolerated by most instruments that use a differential pumping system incorporating diffusion or turbomolecular pumps [6]. However, higher flow rates (i.e., $2 \mathrm{~mL} / \mathrm{min}$ ) can be interfaced with a mass spectrometer by using, for example, an atmospheric pressure source equipped with a heated pneumatic nebulizer and a cryopump system [7]. In situations where the molecular weight of analytes eluting from the HPLC column is sought, the relative success of the analysis depends not only on the efficiency of the pumping system but also on the ability to ionize the polar and labile molecules without excessive fragmentation. In this respect, the choice of the ionization technique used and the extent of internal energy imparted to the analyte also play an impor-

\footnotetext{
*Also at the Department of Chemistry, University of North Carolina at Chapel Hill, Chapel Hill, NC 27514.

Address reprint requests to Pierre Thibault, Atlantic Research Laboratory, National Research Council of Canada, Halifax, Nova Scotia, Canada B3H $3 Z 1$.
}

tant role in observing the parent molecular species. This is especially true for the analysis of thermolabile biomolecules such as peptides.

Among the different direct liquid introduction methods investigated thus far, continuous flow fast atom bombardment (CFFAB) [8] and frit FAB [9] have engendered substantial interest as viable LC/MS interfaces for the analysis of polar and nonvolatile compounds of high molecular weight. This is due in part to the advent of efficient and reliable packed microcolumns $(<400 \mu \mathrm{m}$ i.d.) operating at low flow rates ( $<10 \mu \mathrm{L} / \mathrm{min}$ ). The combination of these microcolumns with "soft" ionization techniques such as FAB permits not only the sensitive detection (typically $<\mathbf{1 0} \mathrm{ng}$ ) of the various protonated molecular ions, but also exploitation of the inherent advantages of microcolumns, namely, high plate numbers $(>50,000$ theoretical plates per meter), high mass sensitivity ( $<10$ pmol per injection), and lower optimum flow rates [10]. Numerous applications using such interfaces have been reported for the analysis of peptide mixtures [11, 12], permethylated oligosaccharides [13], and enzymatic digests of proteins [14-19].

The efficient desorption/ionization of the analyte with the CFFAB interface requires that the aqueous effluent emerging at the end of the probe contain a significant proportion of a suitable FAB matrix (usually $5 \%$ glycerol). This can be achieved by dissolving the matrix in the mobile phase with [20] or without [19] 
splitting the column effluent flow, or by adding the matrix by means of a postcolumn arrangement [21]. The main limitation of the former system is that the addition of the matrix into the LC solvents will change the polarity and viscosity of the mobile phase, which can affect the chromatographic separation. The gradient operation often required to obtain high efficiency separations for a given multicomponent analysis is limited by the solubility of the matrix used to promote the ionization. For example, a 5\% glycerol solution can be dissolved only in an aqueous mobile phase containing a maximum of approximately $60 \%$ acetonitrile. Therefore, separations requiring a higher content of this particular solvent will not be possible. On the other hand, postcolumn addition of the matrix can lead to chromatographic peak broadening, particularly with microbore LC columns [21].

These inconveniences can be avoided by delivering the matrix to the FAB probe tip independently and allowing it to $\mathrm{mix}$ with the LC effluent only at the FAB probe tip. Independent matrix delivery can be achieved by using a coaxial (concentric) capillary arrangement [22-25]. Briefly, this arrangement consists of two coaxial capillaries, the inner one being used to deliver the microcolumn effluent while the annular space between the inner and outer fused-silica capillaries is used to deliver the FAB matrix. Since this system permits mixing of the two effluents only at the probe tip, the extent of chromatographic peak broadening is thereby minimized. In addition, not only can the two flow rates be adjusted independently, but also the matrix composition can be varied without having to modify the chromatographic conditions. This system is ideally suited for use with open tubular liquid chromatography (OTLC) with column inner diameters of $10 \mu \mathrm{m}$ or less and nanoscale packed capillary LC columns (nanoscale with regard to mobile-phase flow rates and sample injection volumes) with column inner diameters of $50 \mu \mathrm{m}$ or less [26], both of which require a make-up flow for FAB desorption/ionization. This coaxial CFFAB interface has also proved to be very useful for the coupling of capillary zone electrophoresis (CZE) with sector mass spectrometry, which offers a high-resolution separation system wilh seleclivity complementary to that of LC $[25,27]$.

To date, the majority of applications of the LC/CFFAB interface have introduced the FAB matrix in the mobile phase. No report thus far has investigated the effects of the FAB matrix on the chromatographic separation. The intent of this work is to compare the effect of adding FAB matrix components (such as glycerol) to the mobile phase of chromatographic systems by employing commercial packed microcolumns (320 $\mu \mathrm{m}$ i.d.) with independent delivery of matrix by means of a coaxial arrangement. This effect is studied for different percentages of glycerol in the LC solvents using only UV detection not connected to the mass spectrometer. Such comparisons are made here for the analysis of synthetic peptide mixtures and tryptic digests. A preliminary account of part of this work has been presented previously [28].

\section{Experimental}

\section{Chemicals}

All peptides used in this study were purchased from Sigma Chemical Co. (St. Louis, MO) and used without further purification. The TPCK trypsin was obtained from Worthington Biochemical Co. (Freehold, NJ). High-performance liquid chromatographic grade acetonitrile, methanol (BDH Chemicals Poole, England), and distilled and deionized (18 Mohm) water (Milli-Q water systems, Millipore Inc., Bedford, MA) were used in the preparation of the samples and mobile phases. All mobile-phase solvents contained $0.1 \%$ trifluoroacetic acid (TFA) (Fisher Scientific, Raleigh, $\mathrm{NC})$ and were filtered $(0.45 \mu \mathrm{m})$ and degassed prior to use.

\section{Tryptic Digests}

Approximately $0.5 \mathrm{mg}$ of the protein was dissolved in $0.5 \mathrm{~mL}$ of a solution of $\mathrm{NH}_{4} \mathrm{HCO}_{3}(0.2 \mathrm{M})$. A solution of $0.5 \mathrm{~mL}$ of TPCK trypsin $(0.4 \mathrm{mg} / 100 \mathrm{~mL}$ in 0.1 $\mathrm{mM} \mathrm{HCl}$ ) was added, and the solution was heated to $37^{\circ} \mathrm{C}$ for $1 \mathrm{~h}$. The tryptic digestion was stopped by freeze-drying the sample and redissolving it in $1 \mathrm{~mL}$ of $\mathrm{H}_{2} \mathrm{O}-0.1 \%$ TFA.

\section{Mass Spectrometry}

All mass spectra were acquired with a VG Analytical ZAB-EQ tandem hybrid mass spectrometer of BEqQ configuration equipped with a VG 11-250J data system. The signals recorded in the conventional mass spectral analyses were detected at the double focus point of the instrument. The mass spectrometer was equipped with an $8-\mathrm{keV}$ Xe beam (Ion Tech FAB gun, 1 mA discharge current; Ion Tech, Middlesex, England), a standard VG CF-FAB source heated at $30-60^{\circ} \mathrm{C}$, and a CFFAB probe using a semihemispherical tip. Conventional $\mathrm{FAB}$ mass spectra were obtained at an accelerating voltage of $8 \mathrm{keV}$ with a static resolution $(10 \%$ valley definition) of approximately 1500 , scanning the range 1600-300 Da with a cycle time of 5-10 s. Owing to fluctuation of the source pressure in experiments using flow rates of 3-4 $\mu \mathrm{L} / \mathrm{min}$, the mass assignment was subject to slight variation from scan to scan; however, the precision on these measurements was reliable to the nearest $\pm 0.5 \mathrm{Da}$.

\section{Liquid Chromatographic Analysis with UV Detection}

The change in chromatographic efficiency of a commercial Alltech $25 \mathrm{~cm} \times 320 \mu \mathrm{m}$ i.d. 5- $\mu \mathrm{m}$ spherical Rosil $C_{18}$ (Eke, Belgium) column, extended by a transfer line of $75-\mathrm{cm}$ fused-silica capillary of $25 \mu \mathrm{m}$ i.d. (150 $\mu \mathrm{m}$ o.d.), with different mobile-phase composi- 


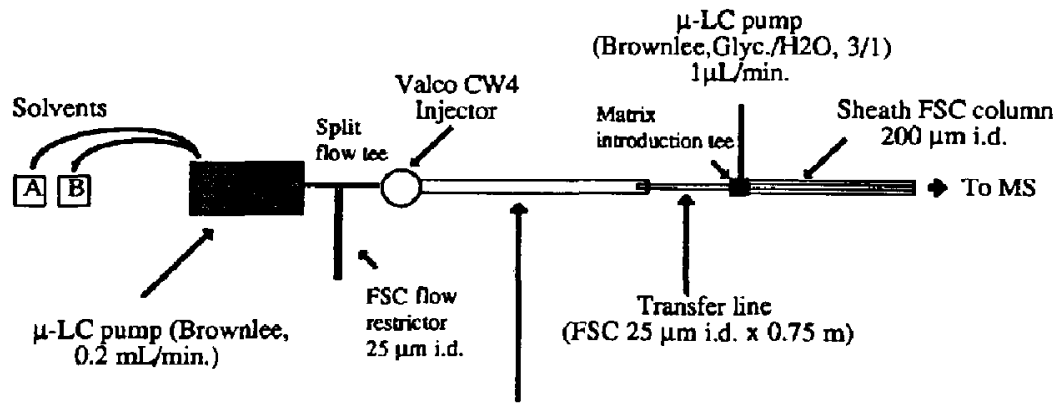

H-column C-18 FSC

(Rosil, $0.32 \mathrm{~mm}$ i.d. $\times 25 \mathrm{~cm}$ )
Figure 1. Schematic representation of the LC/MS interface using a $320 \mu \mathrm{m}$ i.d. $C_{18}$ column (see text for description). tions was studied by using an ISCO $\mu \mathrm{LC}$ UV detector set at $214 \mathrm{~nm}$ (Isco Inc., Lincoln, NE) equipped with a microcell of $0.06 \mu \mathrm{L}$ internal volume. Solvents were delivered with a Brownlee syringe pump (Applied Biosystems, Santa Clara, CA).

\section{Liquid Chromatography Combined with Continuous Flow Fast Atom Bombardment Mass Spectrometry Interface}

The LC/CFFAB/MS interface used in the present study is depicted schematically in Figure 1. This system uses a commercial $320 \mu \mathrm{m}$ i.d. $\mathrm{C}_{18}$ packed microcolumn identical to that described above except that the transfer line now terminates at the end of the FAB probe. The coaxial arrangement, which is a modification of the original interface of de Wit et al. [22], uses a sheath fused-silica capillary ( $200 \mu \mathrm{m}$ i.d., $350 \mu \mathrm{m}$ o.d.) to deliver a solution of glycerol-water $(1: 3)$ at a flow rate of $1 \mu \mathrm{L} / \mathrm{min}$ by using a Brownlee syringe pump (Applied Biosystems). The inner and outer capillaries were coupled together using a 1/16-in. stainless steel tee, and both terminated at the end of the CFFAB probe tip. In experiments in which the FAB matrix was incorporated in the mobile phase, the sheath capillary and the independent matrix delivery system were removed. Injections were made with a Valco CW4 injector with a 0.1$\mu \mathrm{L}$ loop. A column flow rate of $3 \mu \mathrm{L} / \mathrm{min}$ was obtained by splitting the effluent from a Brownlee syringe pump operating at $0.2 \mathrm{~mL} / \mathrm{min}$ by using a stainless steel tee and a fused-silica capillary restrictor.

\section{Results and Discussion}

For purposes of clarity and to differentiate the factors contributing to the overall efficiency of the LC/MS analysis, the effect of glycerol on the chromatographic separation and its possible influence on postcolumn peak broadening are addressed separately in the following discussion.

\section{Effect of Glycerol on Chromatographic Separation}

In the early development of the LC/FAB/MS interface, it was noted that the addition of a FAB matrix to the mobile phase could have some adverse effect on the chromatographic performance of analytical columns [21]. However, no systematic study of this effect has been documented thus far. In consideration of the popularity of CFFAB for the characterization of synthetic peptides and proteolytic fragments of proteins (for which water-acetonitrile solvent systems with gradient elution is the preferred approach), we decided to undertake a study of the effect of varying percentages of glycerol on the retention characteristics of one of the tryptic digests (glucagon) used in the present work, together with simple synthetic peptide mixtures. This study employed a $320 \mu \mathrm{m}$ i.d. packed LC column coupled with a transfer line $(75 \mathrm{~cm} \times 25 \mu \mathrm{m}$ i.d.) (Figure 1). The column effluent was introduced into a UV detector microcell via the transfer line. To maintain a constant concentration of glycerol during gradient elution, the matrix was added to both the aqueous and organic components. Because glycerol is not miscible with pure acetonitrile, only $60 \%$ acetonitrile could be used with a $10 \%$ glycerol solution. The mobile-phase system consisted of an aqueous solvent $\mathrm{A}(0.1 \%$ TFA, $0-10 \%$ glycerol in $100-90 \%$ water) and an organic solvent $\mathrm{B}(0.1 \% \mathrm{TFA}, 40 \%$ aqueous solution containing $0-25 \%$ glycerol in $60 \%$ acetonitrile).

The mobile-phase solvents contained increasing percentages of glycerol $(0,1,2,5$, and $10 \%)$. Starting with glycerol absent from the mobile phase, replicate injections of the glucagon tryptic digest and the peptide mixtures were made for each mobile-phase composition with the UV detector set to $214 \mathrm{~nm}$, After each series of analyses, the mobile-phase reservoirs were replaced and the entire chromatographic system was equilibrated for an hour before the next series of injections.

Small changes in the retention times were observed upon variation of the mobile-phase content of glycerol for any given peptide mixture. These variations were typically less than 2 min over the practical range of the addition of $0-5 \%$ glycerol. It was noted that the retention time and $k^{\prime}$ first decreased from 0 to $2 \%$ glycerol and then progressively increased as the glycerol content increased toward $10 \%$.

More important variations were observed in peak 


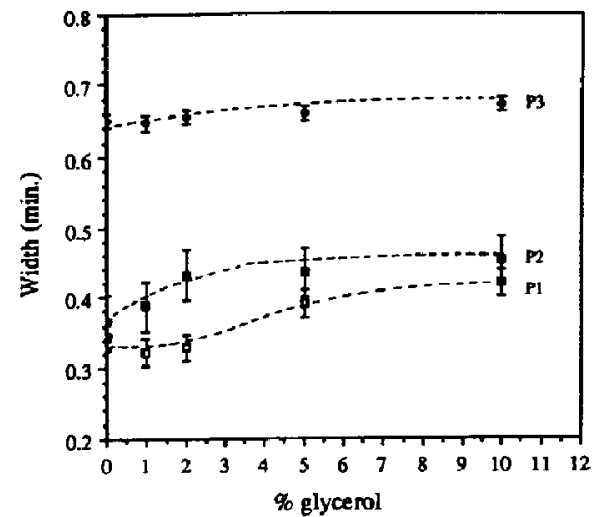

(a)

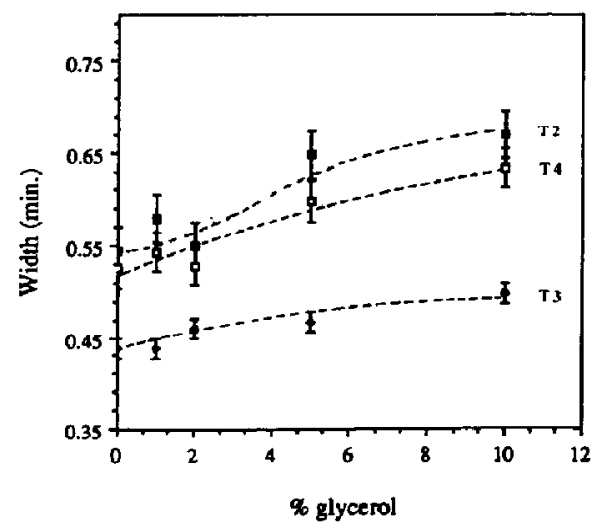

(b)

Figure 2. Influence of glycerol on the column peak width broadening (measured at half-height) in the LC/UV analysis. (a) Analysis of a mixture containing approximately $70 \mathrm{ng}$ of each peptide. Linear gradient from $15 \%$ B to $100 \%$ B in $40 \mathrm{~min}$ (solvent A: $0.1 \%$ TFA, $0-10 \%$ glycerol in $100-90 \%$ water; solvent B: $0.1 \%$ TFA, $40 \%$ aqueous solution containing $0-25 \%$ glycerol in $60 \%$ acetonitrile). Injection of $0.1 \mu \mathrm{L}$ at a flow rate of $3 \mu \mathrm{L} / \mathrm{min}$. (P1, VQAAIDYING; P2, YGGFL; P3, KFLGLM). (b) Analysis of a tryptic digest of 106 pmol of glucagon. Linear gradient from $5 \%$ B to $100 \%$ $B$ in 20 min (T2, fragment 13-17, YLDSR; $T 3$, fragment 19-29, AQDFVQWLMNT; T4, fragment 18-29, RAQDFVQWLMNT).

widths for all the peptide mixtures studied. This is shown graphically in Figure $2 a$ and $b$, which represent the half-height peak width variations as a function of the percentage of glycerol for a mixture of three synthetic peptides and the peptide fragments from a glucagon tryptic digest, respectively. The identification of individual components was obtained by comparing the elution profile with the LC/MS analysis performed on the same samples (described in the following section). The tryptic digestion of glucagon yields at least four peptides, fragments $T_{1}, T_{2}, T_{3}$, and $T_{4}$, in their respective order of elution. Tryptic fragment $T_{1}$ is not included in Figure $2 b$, because the intensity of the corresponding UV absorption signal was so weak that no reliable peak width measurement could be made over the entire range of glycerol addition.
An examination of Figure $2 a$ and $b$ indicates that the peak widths at half height for all peptides progressively increased with higher glycerol concentration. This effect can be translated into an apparent loss of the column separation efficiency, which was, however, regained when the mobile phase was returned to $0 \%$ glycerol. Not all compounds were equally affected by such modification of the mobile-phase composition, but, in general, this variation was most significant from 0 to $5 \%$ glycerol. A comparison of the peak broadening between analyses done at 0 and $5 \%$ glycerol typically showed an enlargement ranging from $2 \%$ to $19 \%$ in the peak width at half height. The width of the peak corresponding to eledoisin-related peptide $P 3$ was less affected by the variation of glycerol over the range studied than, for example, the variation observed for Leu-enkephalin and glucagon tryptic fragment $T_{3}$. This suggests that the effect of the matrix is likely to be dependent on the polarity of the analyte. Peak asymmetry calculations were also performed (data not shown) and showed a similar trend, the peaks becoming more asymmetrical, because of tailing, with the addition of glycerol.

This behavior can be rationalized by considering glycerol at lower concentrations to be acting simply as an alcohol, increasing the polarity of the mobile phase as well as changing its selectivity, and giving rise to the observed retention characteristics. As the percentage of glycerol is increased toward $10 \%$, not only are the chemical properties of the mobile phase changed, but also, more important, its physical properties are modified. The viscosity of the mobile phase increases upon going from 0 to $10 \%$ glycerol. This increase is reflected in a gradual rise in the column back pressure and results in a reduction of the chromatographic separation efficiency. The situation is complicated by the fact that strongly associating solvent mixtures, such as water-acetonitrile and water-methanol, show anomalous variations in viscosity with composition [29], and this could also contribute to the observed variation in the retention characteristics.

\section{Effect of Glycerol as a Mobile Phase Additive in Liquid Chromatography Combined with Mass Spectrometry Analysis}

In consideration of the overall LC/MS analysis, the influence of glycerol on the separation, as discussed above, must be put in perspective with other factors, such as extra length of transfer line and memory effects of the CFFA $B$ probe tip, that may also contribute to peak broadening. For an early-eluting component $\left(k^{\prime}=1\right)$ on a packed microcolumn $25 \mathrm{~cm} \times 320 \mu \mathrm{m}$ i.d. (5 $\mu \mathrm{m}$ particle size), the corresponding column peak volume is typically $0.8 \mu \mathrm{L}$ [30]. The addition of a transfer line with a void volume of $1.5 \mu \mathrm{L}(75 \mathrm{~cm}$ long $\times 25 \mu \mathrm{m}$ i.d.) will contribute to a volume dispersion of $0.04 \mu \mathrm{L}(0.17 \mu \mathrm{L}$ if a $50-\mu \mathrm{m}$ i.d. transfer line is used) [30]. Since the variance of the observed peak 
corresponds to the summation of both the column and transfer line variance, we can thus calculate the ratio of the observed peak volume to the separation column volume. For the above example, the observed peak volume would be $0.2 \%$ larger than the actual separation column peak volume ( $2 \%$ if a $50-\mu \mathrm{m}$ i.d. transfer line was used). Similar increases are to be expected when considering peak widths. Therefore, the effect of peak broadening due to the transfer line is minimal when capillary transfer lines of small inner diameter $(50 \mu \mathrm{m}$ i.d. or less) are used with the $320-\mu \mathrm{m}$ i.d. LC columns and is not likely to account for a large variation of the observed peak width.

More important contributions to the increased peak width involve the influence of wetting and dynamic mixing of the column effluent at the tip of the FAB probe. Although the use of a hemispherical probe tip is reported to reduce the peak broadening over the use of a flat-faced probe tip, diffusion-related memory effects are still likely to occur [31]. In flow injection experiments with amino acids, it was determined that the observed peak width is influenced by the concentration of the analyte. For example, $0.1-\mu \mathrm{L}$ injections of 7,70 , and $700 \mathrm{ng} / \mu \mathrm{L}$ solutions of domoic acid (0.7, 7, and $70 \mathrm{ng}$ injected onto the column) gave peak widths at half height of 6,8 , and $16 \mathrm{~s}$, respectively, using a $3-\mu \mathrm{L} / \mathrm{min}$ flow rate in a $75-\mathrm{cm}$ long $50-\mu \mathrm{m}$ i.d. transfer line. For an ideal injection system free of efficiency losses through bad conncctions, a $0.1-\mu \mathrm{L}$ sample plug should elute with a peak width at half height of less than $3 \mathrm{~s}$. This is obviously not the case here, and the situation is further exacerbated with samples of higher concentration. Similar effects were observed previously on the flow injection analysis of substance $P$ [8]. These results alone indicate that not only is LC/MS peak broadening dependent on the actual column peak volume, but also it is affected to a greater extent by the amount of analyte and by how the analyte is distributed on the probe tip at a given time. Since the ion desorption efficiency is related to the area of sample surface directly exposed to the FAB beam and the requirement of keeping a "wet" probe tip, the actual Einsteinian diffusion of the analyte from the bulk of the drop of FAB matrix to the surface of the drop and the dynamic mixing occurring in the drop of FAB matrix on the FAB probe tip will be partly responsible for the observed peak broadening.

In the LC/CFFAB/MS analysis, this "memory effect" will be superimposed upon the chromatographic peak broadening resulting from the addition of glycerol to the mobile phase. The combined effect will then produce a broader peak width at half height than expected from the column peak volume alone. The overall effect of glycerol on the LC/MS separation was evaluated by repeating the analysis of the two peptide mixtures using the arrangement described in the previous section with the UV detector but with the transfer line now terminating at the end of the CFFAB probe. It should be noted that the extent of chromatographic peak broad- ening due to the addition of the FAB matrix to the solvents (Figure 2) compared to the corresponding variation observed with the mass spectrometer will allow separation of the relative contributions of the two peakbroadening effects.

Figure 3 illustrates the extracted ion chromatograms of the protonated molecular ions of the analysis of three synthetic peptides using glycerol mixed in the mobile phase (Figure 3a) with that of the coaxial arrangement where the matrix is introduced and mixed with the LC effluent only at the probe tip (Figure $3 \mathrm{~b}$ ). The first separation, which uses glycerol mixed in the mobile phases, was obtained by using a linear gradient varying from $15 \%$ to $60 \%$ acetonitrile-water ( $5 \%$ glycerol and $0.1 \%$ TFA present in both solvents) in 40 min for a $0.1-\mu \mathrm{L}$ injection of 60-70 ng of each peptide. It should be mentioned that retention times of each peptide in LC/MS analysis were within $\pm 1.5 \mathrm{~min}$ of those observed using the UV detector (see previous section). This variation is attributed to the slightly different flow rates obtained in the two experiments and emphasizes the difficulty in having reproducible and accurate low flow rates. Nonetheless, comparison

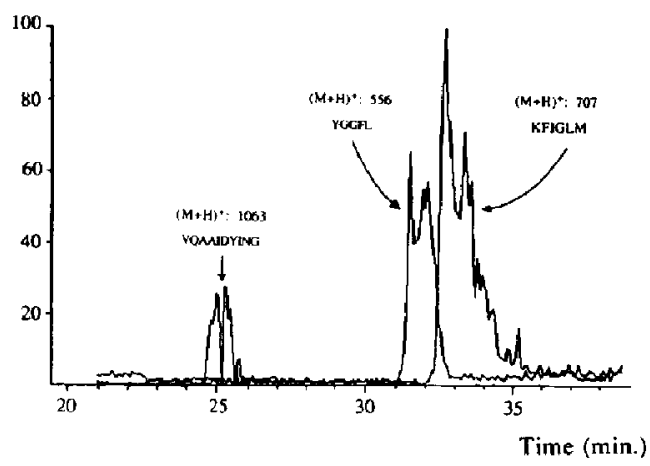

(a)

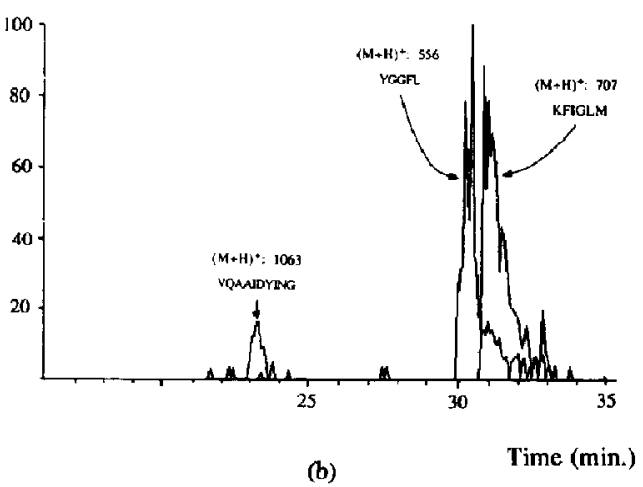

Figure 3. LCICF/FAB analyses of a peptide mixture (decapeptide, Leu-enkephalin, and eledoisin-related peptide) on a 320$\mu \mathrm{m}$ i.d. $\mathrm{C}_{18}$ column. Injection of $0.1 \mu \mathrm{L}$ at a flow rate of $3 \mu \mathrm{L} / \mathrm{min}$. (a) Linear gradient from $15 \%$ B to $100 \%$ B in 40 min (ACN-glycerol-water-TFA; A: 0/5/95/0.1; B: 60/5/35/0.1). (b) Coaxial LC/MS separation using a linear gradient from $15 \%$ B to $65 \%$ B in $40 \mathrm{~min}$ (A: 0/0/100/0.1; B: 100/0/0/0.1). 
between peak widths observed in the LC/MS experiment (Figure 3a) and those reported in Figure 2 for the UV detector with the same mobile-phase composition should still be valid in evaluating the peak-broadening effects discussed above. From duplicate analyses, the mean peak widths at half height for the first three eluting peptides from the precolumn matrix addition configuration (Figure 3a) are $0.87,1.00$, and $1.12 \mathrm{~min}$, respectively. These values are approximately a factor of 2 larger than those reported in Figure 2a. As discussed for the flow injection analysis, the observed increase in peak width in the LC/MS experiment compared to that in the LC/UV analysis is attributed primarily to the sample diffusion at the probe tip (i.e., the memory effect).

On the other hand, when the same analysis is performed with the coaxial LC/MS interface, the chromatographic peak widths at half height are smaller (Figure $3 b$ ) than with the precolumn matrix addition. For the same three peptides, the peak widths at half height are now $0.45,0.68$, and $0.90 \mathrm{~min}$ and correspond to an increase of a factor of only 0.5 , compared with the LC/UV experiment without glycerol in the mobile phase. Therefore, upon going from the LC/MS coaxial interface to the LC/MS precolumn matrix addition configuration, the peak width at half height of these synthetic peptides increases by $25-90 \%$.

The LC/MS analysis of the glucagon tryptic digest (Figure 4) showed even more pronounced peak broadening when glycerol was incorporated in the mobile phase (Figure 4a) as opposed to the coaxial mode of introduction (Figure $\mathbf{4 b}$ ). It should be emphasized again that the variation of retention time observed between the two types of analysis of Figure 4 is due mainly to fluctuations in flow rate arising from the difficulties in obtaining accurate flow rate measurement using a pressure-controlled split system.

Comparison of the different ion chromatograms of Figure $4 a$ and $b$ and also of replicate injections obtained under the same gradient elution indicate that the corresponding peak widths increase by 121 and $76 \%$ for the tryptic fragments 13-17 and 18-29 (T2 and T4, respectively, of Figure $2 \mathrm{~b}$ ). The peak width of the tryptic fragment $19-29$ is marginally affected $(5 \%$ increase from Figure $4 \mathrm{a}$ and $4 \mathrm{~b}$ ) in going from one system to the other. This behavior is consistent with that observed in the LC/UV analysis (Figure $2 b$ ), where this tryptic fragment showed an increase of only $5 \%$ of its peak width when the mobile-phase content of glycerol was varied from 0 to $5 \%$. The other two peptides showed increases of $19-20 \%$ over the same mobile-phase composition. Therefore, the observed peak width can increase by as much as a factor of 2 between the two LC/MS configurations. From both the LC/UV and LC/MS studies, it appears that the extent of this broadening varies with the nature of the analyte, although the underlying reasons for this observation are unknown at present. The fact that the peak enlargement is more pronounced in the LCMS study is due mainly to chromatographic
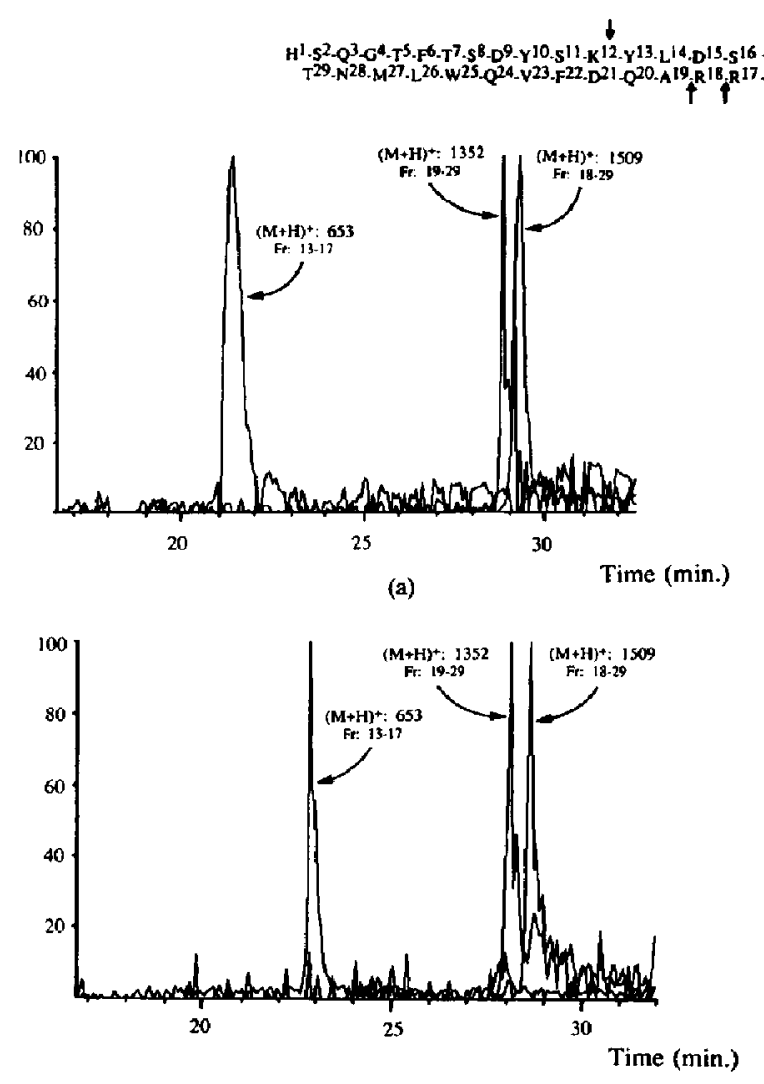

(b)

Figure 4. Selected ion chromatograms corresponding to the LC/CF/FAB analysis of glucagon tryptic digest (106 pmol) using (a) $5 \%$ glycerol in mobile phase. Linear gradient from 5 to $100 \%$ B in $20 \mathrm{~min}$ (ACN-glycerol-water-TFA; A: 0/5/95/0.1; B:60/5/35/0/1). (b) Coaxial LC/MS separation using a linear gradient from $5 \%$ B to $65 \%$ B in $20 \mathrm{~min}$ (A: $0 / 0 / 100 / 0.1 ;$ B: $100 / 0 / 0 / 0.1$ ).

broadening arising from the introduction of the glycerol in the solvents, which is then compounded by the radial diffusion of the analyte at the probe tip.

The difference in flow dynamics at the tip of the FAB probe between the two experimental configurations probably accounts for a significant amount of the observed decrease (5-121\% for glucagon tryptic digest and $25-90 \%$ for the peptide mixture) in chromatographic peak width that occurs upon going from the precolumn addition of glycerol to the coaxial configuration. As discussed earlier, we think that diffusion of the analyte from the bulk of the homogeneous matrix droplet is responsible for the observed peak broadening in the precolumn matrix addition configuration. In contrast, the coaxial interface yields a heterogenous flow stream, where the LC effluent is constrained to the center of the flow stream and actively transported to the surface of the FAB droplet where ion desorption occurs.

Although the preceding discussions have focused on the changes in chromatographic peaks in terms of 


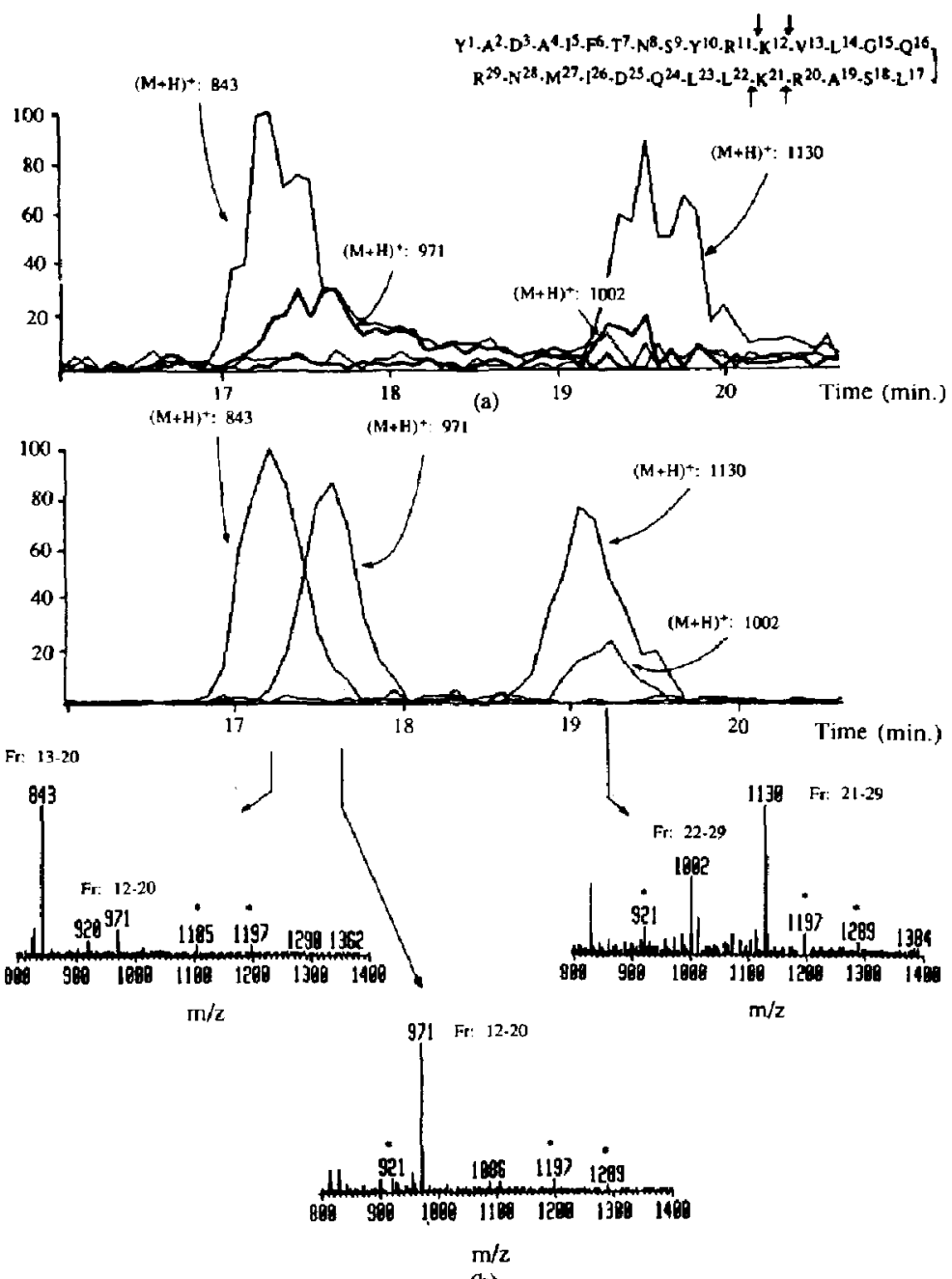

(b)
Figure 5. LC/CFFAB analysis of bovine growth hormone releasing factor 1-29 tryptic digest (41 pmol) using (a) 5\% glycerol in mobile phase. Linear gradient from $0 \%$ B to $100 \%$ B in $20 \mathrm{~min}$ (ACN-glycerol-water-TFA; A: $0 / 5 / 95 / 0.1 ; B: 60 / 5 / 35 / 0.1)$. (b) Coaxial LC MS separation using a linear gradient from $0 \%$ $\mathrm{B}$ to $100 \% \mathrm{~B}$ in $20 \mathrm{~min}$ (A: 0/0/100/0.1; B: $100 /$ $0 / 0 / 0.1$ ) with selected mass spectra (single scan) corresponding to different tryptic fragmenls. ("indicales matrix peaks.) peak width, the resultant effect of this is directly reflected by the chromatographic separation efficiency. In the conventional formula for the calculation of the number of theoretical plates, the number of plates is inversely proportional to the square of the half-height peak width for a given retention time. It can be deduced that an increase in peak width of $20 \%$, such as in the case of the LCJUV experiment, will lead to a decrease in separation efficiency by a factor of 1.4. However, this effect is more important for the LC/MS experiment where an increase in peak width of a factor of 2 as noted between the two types of interface involves a factor of 4 loss of separation efficiency.

The fact that peak broadening is reduced in the coaxial configuration offers a potential advantage in sensitivity over the LCMS configuration. Previous studies [23] indicated that lower detection limits can be achieved with this interface. Although the present work did not focus on establishing detection limits for both LC/MS configurations, it can be appreciated from
Figures 3 and 4 that better signal-to-noise (S/N) ratios are obtained when using the coaxial interface. On replicate injections of the different peptide mixtures of Figures 3 and 4 , it was found that the $S / N$ ratio of the coaxial interface was generally better by a factor of 2 compared to the other LC/MS configuration.

The advantages of this interface are further demonstrated in Figure 5 for the LC/MS analysis of tryptic fragments of bovine growth hormone-releasing factor, a peptide containing 29 amino acid residues. The analysis performed with glycerol in the mobile phase is illustrated in Figure 5a for a linear gradient of 0-60\% acetonitrile-water (5\% glycerol and $0.1 \%$ TFA in both solvents) and can be compared to the analysis obtained from the coaxial interface (Figure 5b) using a $0-100 \%$ gradient of acetonitrile-water (0.1\% TFA). As observed in Figure 5a, owing to the relatively wide compositional change (0-60\% organic solvent) combined with the relatively low concentration of glycerol, a stable matrix surface on the probe tip was not maintained 
throughout the entire gradient profile. This caused ion source pressure fluctuations, which affect the ion source high voltage and caused erratic signal intensities in the tryptic fragment's ion chromatograms. In contrast, the coaxial CFFAB configuration gave good $\mathrm{S} / \mathrm{N}$ ratios for all the peptides as indicated by the quality of the selected ion traces and their corresponding mass spectra (Figure 5b). The stability of the ion current also allows better definition of a consistent background spectrum, which in turn permits extraction of a mass spectrum of each peptide exempt of matrix contribution.

\section{Conclusions}

The use of packed fused-silica capillary columns in conjunction with a coaxial CFFAB interface has been shown to be an effective technique for the analysis of peptide mixtures by mass spectrometry. This arrangement offers several advantages over the use of a glycerol matrix in the mobile phase. The independent delivery of the $F A B$ matrix to the probe tip significantly decreases the chromatographic peak broadening and tailing found when the matrix is added to the mobile phase. In addition, the coaxial interface further improved separation efficiency by reducing peak broadening attributed to diffusional "memory effects," owing to the improved flow dynamics of the analyte at the probe tip. For the peptide mixtures analyzed in this investigation, including those originating from tryptic digestion, it was observed that the use of a coaxial arrangement reduces the observed peak width by $5-155 \%$ with respect to a similar gradient elution system containing a 5\% glycerol solution. The extent of the overall peak broadening, however, depends on the nature of the compound. A direct advantage of the narrower peak shape in coaxial CFFAB is that a higher flux of analyte reaches the probe tip over a given time.

In addition to minimizing chromatographic peak broadening, this interface also allows variation of the matrix composition and concentration without compromising the separation and/or requiring a reoptimization of the chromatographic conditions.

\section{References}

1. Games, D. E. Adv. Chromatogr. 1983, 21, 1.

2. Edmonds, C. G.; McCloskey, J. A.; Edmonds, V. A. Biomed. Mass Spectrom. 1983, 10, 277.
3. Vestal, M. L. Science 1984, 19, 275.

4. Bruins, A. P. J. Chromatogr. 1985, 323, 99.

5. Niessen, W. M. A. Chromatographia 1986, 21, 277.

6. Arpino, P. J.; Guiochon, G.; Krien, P.; Devant, G. J. Chromatogr. 1979, 185, 529.

7. Covey, R. R.; Lee, E. D.; Bruins, A. P.; Henion, J. D. Anal. Chem. 1986, 58, 1451a.

8. Caprioli, R. M.; Fan, T.; Cottrell, J. S. Anal. Chem. 1986, 58, 2949.

9. Ito, Y.; Takeuchi, T.; Ishii, D.; Goto, M. J. Chromatogr. 1985, $346,161$.

10. Verzele, M.; Dewaele, C.; De Weerdt, M. LC-GC 1988, 6, 966.

11. Ito, Y.; Takeuchi, T.; Ishii, D.; Goto, M.; Miztuno, T. I. Chromatogr. 1986, 358, 201.

12. Ashcroft, A. E. Org. Mass Spectrom. 1987, 22, 754.

13. Boulenguer, P.; Leroy, U.; Alonzo, J. M.; Montreuil, J.; Ricart, G.; Colbert, C.; Duquet, D.; Dewaele, C.; Fournet, B. Anal. Biochem. 1988, 168, 164.

14. Caprioli, R. M.; Moore, W. T.; Fan, T. Rapid Commun. Mass Spectrom. 1987, 1, 15.

15. Hutchison, D. W.; Woolfitt, A. R.; Ashcroft, A. E. Org. Mass Spectrom. 1987, 22, 394.

16. Ashcroft, A. E.; Chapman, J. R.; Cottrell, J. S. J. Chromatogr. 1987, 394, 15.

17. Caprioli, R. M. Biomed. Environ. Mass Spectrom. 1988, 16, 35.

18. Caprioli, R. M.; DaGue, B.; Wilson, K. I. Chromatogr. Sci. $1988,26,640$.

19. Caprioli, R. M.; Moore, W. T.; DaGue, B.; Martin, M. 7. Chromatogr. 1988, 443, 355.

20. Capriuli, R. M.; Da Gue, B.; Fanl, T.; Muore, W. T. Büctem. Biophys. Res. Commun. 1987, 146, 291.

21. Games, D. E.; Pleasance, S.; Ramsey, E. D.; McDowell, M. A. Biomed. Environ. Mass Spectrom. 1988, 15, 179.

22. de Wit, J. S. M.; Deterding, L. J.; Moseley, M. A.; Tomer, K. B.; Jorgenson, J. W. Rapid Commun. Mass Spectrom. 1988, $2,100$.

23. Moseley, M. A.; Deterding, L. J.; de Wit, J. S. M.; Tomer, K. B.; Kennedy, R. T.; Bragg, N.; Jorgenson, J. W. Anal. Chem. $1989,61,1577$.

24. Deterding, L. J.; Moseley, M. A.; Tomer, K. B.; Jorgenson, J. W. Anal. Chem. 1989, 61, 2504.

25. Moseley, M. A.; Deterding, L. J.; Tomer, K. B.; Jorgenson, J. W. J. Chromatogr. 1989, 408, 197.

26. Jorgenson, J. W.; Guthrie, E. J. J. Chromatogr. 1983, 255, 335.

27. Moseley, M. A.; Deterding, L. J.; Tomer, K. B.; Jorgenson, J. W. Rapid Commun. Mass Spectrom. 1989, 3, 87.

28. Deterding, L. J.; Jorgenson, J. W.; Moseley, M. A.; Pleasance, S.; Thibault, P.; Tomer, K. B. Proceeding's of the 37th ASMS Conference, Miami Beach, FL, 1989; p 1006.

29. Abbot, S. R.; Achener, P.; Berg, J. R.; Stephenson, R. L. J. Chromatogr. 1976, 126, 421.

30. Saito, M.; Hibi, K.; Ishii, D.; Takeuchi, T. In Introduction to Microscale High Performance Liquid Chromatography, Ishii, D., Ed.; VCH: New York, 1988; p 26.

31. Pleasance, S. PhD Dissertation, 1988, University College, Cardiff, U.K. 